\title{
BOUNDS FOR PRIME SOLUTIONS OF SOME DIAGONAL EQUATIONS. II
}

\author{
MING-CHIT LIU
}

\begin{abstract}
Let $b_{j}$ and $m$ be certain integers. In this paper we obtain a bound for prime solutions $p_{j}$ of the diagonal equations of order $k, b_{1} p_{1}^{k}+\cdots+b_{s} p_{s}^{k}=m$. The bound obtained is $C^{(\log B)^{2}}+C|m|^{1 / k}$ where $B=\max _{j}\left\{e,\left|b_{j}\right|\right\}$ and $C$ are positive constants depending at most on $k$.
\end{abstract}

1. Introduction. Throughout $p$ denotes a prime number and $k \geqslant 2$ is an integer. Let $\theta \geqslant 0$ be the largest integer such that $p^{\theta}$ divides $k$. We write $p^{\theta} \| k$. Let

$$
s_{0}= \begin{cases}3 k-1 & \text { if there is a } p \text { satisfying } p \mid k \text { and } k=((p-1) / 2) p^{\theta} \\ 2 k & \text { otherwise. }\end{cases}
$$

$$
s_{1}= \begin{cases}2^{k}+1 & \text { if } 2 \leqslant k \leqslant 11 \\ 2 k^{2}(2 \log k+\log \log k+2.5)-1 & \text { if } k \geqslant 12 .\end{cases}
$$

In this paper we shall prove

THEOREM 1 . Let $b_{1}, \ldots, b_{s}$ be any nonzero integers which do not have the same sign. Let $m$ be any integer satisfying

$$
\sum_{j=1}^{s} b_{j} \equiv m \quad(\bmod K) .
$$

If $s$ is the least integer with $s \geqslant s_{1}$ and if no prime can divide more than $s-s_{0} b_{j}$ then there are constants $C_{j}(k)$ depending on $k$ only such that the equation

$$
\sum_{j=1}^{s} b_{j} p_{j}^{k}=m
$$

Received by the editors November 19, 1984 .

1980 Mathematics Subject Classification (1985 Revision). Primary 10J15; Secondary 10B15.

Key words and phrases. Bounds for prime solutions, diagonal equations, trigonometric sums, Dirichlet's characters, the Hardy-Littlewood method. 
always has a solution in odd primes $p_{j}$ satisfying

$$
\max _{1 \leqslant j \leqslant s} p_{j}<C_{1}|m|^{1 / k}+C_{2}^{(\log B)^{2}}
$$

where $B=\max \left\{\left|b_{1}\right|, \ldots,\left|b_{s}\right|, e\right\}$.

Investigations on bounds for integral solutions of diagonal equations similar to type (1.6) were made by Cassels [3], Birch and Davenport [2], Pitman and Ridout [11], Pitman [12]. On the other hand, results on bounds for prime solutions of (1.6) were obtained by Baker [1] and the author [9]. In all previous works on prime solutions, bounds obtained are of the form $C(k, \delta)^{\left(\max \left|b_{j}\right|\right)^{\delta}}$ for any $\delta>0$. So (1.7) in Theorem 1 gives an essentially better bound than the previous one $[9,(1.6)]$ and our Theorem 1 improves Theorem 1 in [9]. The new bound, $C^{(\log B)^{2}}$ is obtained by using [5, Theorem 6] a zero density estimate for $L$-functions which, as a consequence, replaces the Siegel-Walfisz theorem on prime distribution applied in both [1, Lemma 1] and [9, Lemma 6]. By this zero density estimate we can obtain a better error estimate as shown in our Lemma 2 which enables us to treat terms belonging to category (A) in $\S 4$ below. This change causes not only an improvement on the bound but also a greatly different emphasis in methods.

By (1.1) and (1.2) we see that the divisibility condition on $b_{j}$ in Theorem 1 is better than (for $k \geqslant 4$ ) the condition, $\left(b_{j}, b_{l}\right)=1$ for $j \neq l$, which is usually assumed in additive problems involving primes. By (1.4) and (1.5) our condition on $\mathrm{m}$ coincides with that in the Waring-Goldbach problem [7, p. 100 and p. 108] where the case $b_{j}=1$ was considered.

2. Notation. Throughout we assume that $N$ satisfies

$$
\log N \geqslant N_{0}(\log B)^{2}
$$

where $N_{0}>0$ is a large constant depending on $k$ only.

$\chi(\bmod q)$ denotes a Dirichlet character and $\chi_{0}(\bmod q)$ denotes the principal character. $\chi^{*}(\bmod r)$ is a primitive character, $\tilde{\chi}(\bmod \tilde{r})$ is the exceptional primitive character and $\tilde{\beta}$ is the exceptional zero (see Lemma 1 below). Throughout the constants $c_{j}$ and all implicit constants in the Vinogradov symbols $\ll$, the $O$ symbols are positive and depend at most on $k$. The constants $A_{j}$ are positive absolute. $\phi(q)$ is the Euler function and for real $\alpha$ write $e(\alpha)=\exp (i 2 \pi \alpha)$. Let

$$
P=P(N)=\exp \left(\sqrt{A_{1} \log N} / 10\right), \quad Q=N^{k} P^{-1},
$$

where $A_{1}$ is given in Lemma 1 . The constant $\sqrt{A_{1}} / 10$ in (2.2) will be needed in the proof of Lemma 2. Let

$$
\begin{gathered}
W(a, \chi)=\sum_{n=1}^{q} \chi(n) e\left(\frac{a n^{k}}{q}\right), \\
S(b \alpha)=\sum_{G<p \leqslant N} \log p e\left(b \alpha p^{k}\right), \quad S(b \alpha, \chi)=\sum_{G<p \leqslant N} \chi(p) \log p e\left(b \alpha p^{k}\right),
\end{gathered}
$$

where

$$
G=N\left(6^{k} s|b|\right)^{-1 / k}
$$


For $1 \leqslant a \leqslant q \leqslant P,(a, q)=1$ let $\mathscr{M}(q, a)$ be the major arc which is the set of real $\alpha$ satisfying $|\alpha-a / q| \leqslant \delta_{q}$ with

$$
\delta_{q}=(q Q)^{-1} \text {. }
$$

These major arcs are disjoint. Let $\mathscr{M}$ be the union of all major arcs and $m$ denote minor arcs which is the complement of $\mathscr{M}$ with respect to the set of $\alpha$ satisfying $Q^{-1} \leqslant \alpha \leqslant 1+Q^{-1}$.

For $\alpha \in \mathscr{M}(q, a)$ write $\alpha=a / q+\eta$. If $p>P$ then $(q, p)=1$, since $q \leqslant P$. It follows from the orthogonal relation of characters that

$$
S(b \alpha)=\phi(q)^{-1} \sum_{\chi} W(a b, \bar{\chi}) S(b \eta, \chi) .
$$

Note that if $p>P$ then

$$
S(b \eta, \chi)=S\left(b \eta, \chi^{*}\right)
$$

where $\chi^{*}(\bmod r)$ induces $\chi(\bmod q)$. Put

$$
\left\{\begin{array}{l}
I(b \eta)=\sum_{|b| G^{k}<n \leqslant|b| N^{k}} e( \pm \eta n) n^{-1+1 / k}\left(k|b|^{1 / k}\right)^{-1}, \\
\tilde{I}(b \eta)=-\sum_{|b| G^{k}<n \leqslant|b| N^{k}} e( \pm \eta n) n^{-1+\tilde{\beta} / k}\left(k|b|^{\tilde{\beta} / k}\right)^{-1}
\end{array}\right.
$$

where \pm denotes the sign of $b . \tilde{I}(b \eta)$ is defined only if there is $\tilde{\beta}$. Let

$$
\Delta(b \eta, \chi)= \begin{cases}S\left(b \eta, \chi_{0}\right)-I(b \eta) & \text { if } \chi=\chi_{0}, \\ S\left(b \eta, \tilde{\chi} \chi_{0}\right)-\tilde{I}(b \eta) & \text { if } \chi=\tilde{\chi} \chi_{0}, \\ S(b \eta, \chi) & \text { if } \chi \neq \chi_{0} \text { and } \chi \neq \tilde{\chi} \chi_{0}\end{cases}
$$

By (2.5) we have

$$
\Delta(b \eta, \chi)=\Delta\left(b \eta, \chi^{*}\right)
$$

\section{Lemmas.}

Lemma 1. Let $z=\sigma+i$. There is $A_{1}$ such that the Dirichlet L-function $L\left(z, \chi^{*}\right) \neq 0$ whenever $\sigma \geqslant 1-A_{1} / \log (P(|t|+2))$ for all primitive characters $\chi^{*}(\bmod r)$ and $r \leqslant P$ with the possible exception of at most one primitive character, $\tilde{\chi}(\bmod \tilde{r})$. If there is such an exceptional character then it is quadratic and the unique exceptional zero $\tilde{\beta}$ of $L(z, \tilde{\chi})$ is real and simple and satisfies

$$
A_{2} / \tilde{r}^{1 / 2}(\log \tilde{r})^{2} \leqslant 1-\tilde{\beta} \leqslant A_{1} / \log P .
$$

Proof. See [4, §14].

LEMMA 2. For any real $\lambda \geqslant 1$ we have

$$
\sum_{r \leqslant P} \sum_{\chi^{*}}\left(\int_{-\delta_{r}}^{\delta_{r}}\left|\Delta\left(b \eta, \chi^{*}\right)\right|^{\lambda} d \eta\right)^{1 / \lambda} \ll|b| N^{1-k / \lambda} P^{-2}
$$

where the summation $\Sigma_{\chi^{*}}$ is taken over all $\chi^{*}(\bmod r)$. 
PROOF. The proof is essentially the same as Theorem 7 [5]. In the proof we apply Theorem 6 [5] and put the $T$ there to be $P^{7}$.

Lemma 3. Let $q=q_{1} \cdots q_{t}$ with $\left(q_{j}, q_{l}\right)=1$ for $j \neq l$. Let $\chi(\bmod q)$ be factorized into $\prod_{j=1}^{t} \chi_{j}\left(\bmod q_{j}\right)$. If $(a, q)=1$ then there exist uniquely $a_{j}\left(\bmod q_{j}\right)$ with

$$
\left(a_{j}, q_{j}\right)=1 \quad(j=1, \ldots, t), a=\sum_{j=1}^{t} \frac{a_{j} q}{q_{j}}
$$

and

$$
W(a b, \chi)=\prod_{j=1}^{t} W\left(a_{j} b, \chi_{j}\right) .
$$

Proof. This is essentially Theorem 4.1 in [8, p. 159].

LEMMA 4. Let $h_{1}=h /(h, q)$ and $q_{1}=q /(h, q)$. Let $\chi^{*}(\bmod r)$ induce $\chi(\bmod q)$. Then

$$
W(h, \chi)= \begin{cases}0 & \text { if } r+q_{1}, \\ \phi(q) \phi\left(q_{1}\right)^{-1} W\left(h_{1}, \chi_{1}\right) & \text { if } r \mid q_{1} \text { where } \chi_{1}\left(\bmod q_{1}\right) \\ & \text { is induced by } \chi^{*}(\bmod r)\end{cases}
$$

REMARKs. Lemma 4 is parallel to the known result on the Ramanujan sum and its generalization [6, p. 450]. In fact, we can also prove that $W(h, \chi)=0$ if $r \mid q_{1}$ and $\left(r, q_{1} / r\right)+k$.

Proof. Write $q_{2}=q / q_{1}$ and $n=u q_{1}+v$ with $u=0,1, \ldots, q_{2}-1 ; v=1$, $2, \ldots, q_{1}$. Then

$$
\sum_{n=1}^{q} \chi(n) e\left(\frac{h n^{k}}{q}\right)=\sum_{\substack{v=1 \\\left(v, q_{1}\right)=1}}^{q_{1}} e\left(\frac{h_{1} v^{k}}{q_{1}}\right) T(v)
$$

where $T(v)=\sum_{u=1}^{q_{2}} \chi\left(u q_{1}+v\right)$.

Let $r+q_{1}$. By the same argument as in showing $S(v)=0$ in $[4$, p. 66] we can prove that $T(v)=0$ and hence $W(h, \chi)=0$.

Next consider $r \mid q_{1}$. Let $d=\prod_{p \mid q_{2}, p+q_{1}} p$ and $\mathscr{J}=\left\{u q_{1}+v: 1 \leqslant u \leqslant q_{2}\right\}$. If $\left(v, q_{1}\right)=1$ then

$$
\sum_{\substack{j \in \mathscr{J} \\(j, q)=1}} 1=\sum_{j \in \mathscr{J}} \sum_{n \mid(j, d)} \mu(n)=\sum_{n \mid d} \frac{\mu(n) q_{2}}{n}=q_{2} \prod_{p \mid d}\left(1-p^{-1}\right) .
$$

It follows from $\chi^{*}\left(u q_{1}+v\right)=\chi^{*}(v)$ and (3.4) that if $\left(v, q_{1}\right)=1$ then

$$
T(v)=\chi^{*}(v) \quad \sum_{\substack{u=1 \\\left(u q_{1}+v, q\right)=1}}^{q_{2}} 1=\chi^{*}(v) \phi(q) \phi\left(q_{1}\right)^{-1} .
$$

By (3.3) this proves Lemma 4. 
Lemma 5. (a) If $(a, p)=1$ and $p^{\prime}$ is the modulus of $\chi$ then $|W(a, \chi)| \leqslant 2 k p^{1 / 2}$.

(b) If $(a, q)=1$ and $q$ is the modulus of $\chi$ then for any $\varepsilon>0$ there is a positive constant $C(\varepsilon, k)$ depending at most on $\varepsilon, k$ such that

$$
|W(a b, \chi)| \leqslant C(k, \varepsilon)(q, b)^{1 / 2} q^{1 / 2+\varepsilon} .
$$

PROOF. Part (a) follows from a similar argument as part 1 of the proof of Lemma 8.5 [7].

(b) Let $\chi^{*}(\bmod r)$ induce $\chi(\bmod q), q^{\prime}=q /(b, q), b^{\prime}=b /(b, q)$. Suppose that $r \mid q^{\prime}$. Put $q^{\prime}=\prod_{j=1}^{t} p_{j}^{l_{j}}$ and factorize $\chi^{\prime}\left(\bmod q^{\prime}\right)$ into $\prod_{j=1}^{t} \chi_{j}\left(\bmod p_{j}^{l_{j}}\right)$, where $\chi^{\prime}$ $\left(\bmod q^{\prime}\right)$ is induced by $\chi^{*}(\bmod r)$. Then by Lemmas 4,3 , and Lemma $5(\mathrm{a})$

$$
\begin{aligned}
|W(a b, \chi)| & =\phi(q) \phi\left(q^{\prime}\right)^{-1}\left|W\left(a b^{\prime}, \chi^{\prime}\right)\right| \leqslant(b, q) \prod_{j=1}^{t}\left|W\left(a_{j} b^{\prime}, \chi_{j}\right)\right| \\
& \leqslant(b, q)^{1 / 2}\left((b, q) q^{\prime}\right)^{1 / 2} \prod_{j=1}^{t} 2 k .
\end{aligned}
$$

This proves Lemma $5(\mathrm{~b})$.

4. Major arcs. I. Write

$$
\left\{\begin{array}{l}
\mathscr{W}_{j}=\phi(q)^{-1} \sum_{\chi} W\left(a b_{j}, \bar{\chi}\right) \Delta\left(b_{j} \eta, \chi\right), \\
\mathscr{I}_{j}=\phi(q)^{-1} I\left(b_{j} \eta\right) W\left(a b_{j}, \chi_{0}\right) \\
\tilde{\mathcal{I}}_{j}=\phi(q)^{-1} \tilde{I}\left(b_{j} \eta\right) W\left(a b_{j}, \tilde{\chi} \chi_{0}\right),
\end{array}\right.
$$

where $\tilde{\mathscr{I}}_{j}$ is defined only when the exceptional character exists. By (2.4), (2.7) we have

$$
\begin{aligned}
R_{1}(m) & =\sum_{q \leqslant P} \sum_{a}^{\prime} \int_{-\delta_{q}}^{\delta_{q}} e\left(-m\left(\frac{a}{q}+\eta\right)\right) \prod_{j=1}^{s} S\left(b_{j} \alpha\right) d \eta \\
& =\sum_{q \leqslant P} \sum_{a}^{\prime} e\left(\frac{-m a}{q}\right) \int_{-\delta_{q}}^{\delta_{q}} e(-m \eta) \prod_{j=1}^{s}\left(\mathscr{W}_{j}+\mathscr{I}_{j}+\tilde{\mathscr{I}}_{j}\right) d \eta
\end{aligned}
$$

where the sum $\Sigma_{a}^{\prime}$ is taken over all $a$ with $1 \leqslant a \leqslant q$ and $(a, q)=1$.

There are two categories of terms in the last product of (4.2), namely, (A) terms having at least a factor $\mathscr{W}_{j}$; (B) terms having no factor $\mathscr{W}_{j}$. We shall treat category (A) in this section and category (B) in $\$ 6$.

Let $\mathscr{I}_{j}^{\prime}$ denote either $\mathscr{\mathscr { I }}_{j}$ or $\tilde{\mathscr{I}}_{j}$. In category (A) for each fixed $h=1,2, \ldots, s$ we choose $\prod_{j=1}^{h} \mathscr{W}_{j} \Pi_{j=h+1}^{s} \mathscr{I}_{j}^{\prime}$ as the representative of those terms having exactly $h$ factors $\mathscr{W}_{j}$. Put

$$
T_{h}(m)=\sum_{q \leqslant P} \sum_{a}^{\prime} e\left(\frac{-m a}{q}\right) \int_{-\delta_{q}}^{\delta_{q}} \prod_{j=1}^{h} \mathscr{W}_{j} \prod_{j=h+1}^{s} \mathscr{I}_{j}^{\prime} e(-m \eta) d \eta
$$

Let

$$
(h=1, \ldots, s) \text {. }
$$

$$
\begin{gathered}
\chi_{j}^{\prime}(\bmod q)=\chi_{0}(\bmod q) \text { or } \tilde{\chi} \chi_{0}(\bmod q) \\
I^{\prime}\left(b_{j} \eta\right)=I\left(b_{j} \eta\right) \text { or } \tilde{I}\left(b_{j} \eta\right)
\end{gathered}
$$


Then by Schwarz's inequality and (4.1), (4.3) we have (4.6)

$$
\begin{aligned}
\left|T_{h}(m)\right| \leqslant & \sum_{p \leqslant P} \phi(q)^{-s} \sum_{\substack{\chi_{j} \\
j=1, \ldots, h}}\left|\sum_{a}^{\prime} e\left(\frac{-m a}{q}\right) \prod_{j=1}^{h} W\left(a b_{j}, \bar{\chi}_{j}\right) \prod_{j=h+1}^{s} W\left(a b_{j}, \chi_{j}^{\prime}\right)\right| \\
& \times \prod_{j=1}^{h}\left(\int_{-\delta_{q}}^{\delta_{q}}\left|\Delta\left(b_{j} \eta, \chi_{j}\right)\right|^{n_{1}} d \eta\right)^{1 / n_{,}} \prod_{j=h+1}^{s}\left(\int_{-\delta_{q}}^{\delta_{q}}\left|I^{\prime}\left(b_{j} \eta\right)\right|^{n_{j}} d \eta\right)^{1 / n_{j}},
\end{aligned}
$$

where $\sum_{\chi, j=1, \ldots, h}$ denotes $h$ summations each of which is taken over all $\chi(\bmod q)$ and $n_{j} \geqslant 1$ are integers satisfying $\sum_{j=1}^{s} 1 / n_{j}=1$. Note that each $\chi_{j}(\bmod q)$ is induced by a unique $\chi_{j}^{*}\left(\bmod r_{j}\right)$ with $r_{j} \mid q$ and that each $\chi_{j}^{*}\left(\bmod r_{j}\right)$ and each $q$ with $r_{j} \mid q$ induce a unique $\chi(\bmod q)$. Then by $(2.8),(4.6)$ we have

$$
\begin{aligned}
\left|T_{h}(m)\right| \leqslant & \sum_{\substack{r_{j} \leqslant P \\
j=1 \ldots \ldots h}} \sum_{\chi_{j}^{*}}\left\{\sum_{\substack{q=1 \\
r_{j} \mid q, j=1 \ldots, h}}^{\infty} \phi(q)^{-s}\left|\sum_{a}^{\prime} e\left(\frac{-m a}{q}\right) \prod_{j=1}^{h} W\left(a b_{j}, \chi_{0} \bar{\chi}_{j}^{*}\right)\right|\right. \\
& \left.\times \prod_{j=h+1}^{s} W\left(a b_{j}, \chi_{j}^{\prime}\right) \mid\right\} \\
& \times \prod_{j=1}^{h}\left(\int_{-\delta_{r_{j}}}^{\delta_{r_{r}}}\left|\Delta\left(b_{j} \eta, \chi_{j}^{*}\right)\right|^{n_{\prime}} d \eta\right)^{1 / n_{j}} \prod_{j=h+1}^{s}\left(\int_{-\delta_{1}}^{\delta_{1}}\left|I^{\prime}\left(b_{j} \eta\right)\right|^{n_{j}} d \eta\right)^{1 / n_{1}} .
\end{aligned}
$$

By Lemma 5 with $\varepsilon=(10 s)^{-1}$, the infinite sum inside the curly brackets of (4.7) is

$$
\ll \sum_{q=1}^{\infty} \phi(q)^{-s+1} \prod_{j=1}^{s}\left|b_{j}\right|^{1 / 2} q^{1 / 2+1 / 10 s} \ll B^{s / 2}
$$

since by (1.2) we have $s \geqslant 5$ for any $k \geqslant 2$. Also by (2.6) we have $I^{\prime}\left(b_{j} \eta\right) \ll N$ and then by (2.3), (2.2)

$$
\left(\int_{-\delta_{1}}^{\delta_{1}}\left|I^{\prime}\left(b_{j} \eta\right)\right|^{n_{j}} d \eta\right)^{1 / n_{1}} \ll N^{1-k / n_{j}} P^{1 / n_{j}}
$$

It follows from (4.7), (4.8), (4.9) and Lemma 2 that

$$
\begin{aligned}
T_{h}(m) & \ll B^{s / 2}\left(\prod_{j=1}^{h}\left|b_{j}\right| N^{1-k / n_{j}} P^{-2}\right)\left(\prod_{j=h+1}^{s} N^{1-k / n_{j}} P^{1 / n_{j}}\right) \\
& \ll B^{3 s / 2} N^{s-k} P^{-1}=E_{1}, \quad \text { say },
\end{aligned}
$$

since $\sum_{j=1}^{s} 1 / n_{j}=1$.

\section{Singular series.}

LeMma 6. For a given $p$ let $p^{\theta} \| k$ and $p^{\phi} \| b$. Suppose that $p^{t}$ and $p^{j}$ are the moduli of $\chi_{0}$ and $\chi_{1}$ respectively and

$$
u=2 \phi+\theta+ \begin{cases}3 & \text { if } p=2 \\ 1 & \text { if } p \geqslant 3\end{cases}
$$


If $1 \leqslant j \leqslant u-2 \phi-\theta, t \geqslant u+1$ and $(a, p)=1$ then

$$
W\left(a b, \chi_{0}\right)=W\left(a b, \chi_{1} \chi_{0}\right)=0 .
$$

Proof. The proof is essentially the same as Lemma 1 [9].

Lemma 7. Let $q=q_{1} q_{2},\left(q_{1}, q_{2}\right)=1$ and factorize $\chi_{j}(\bmod q)$ into $\prod_{l=1}^{2} \chi_{j l}$ $\left(\bmod q_{l}\right)(j=1,2, \ldots, s)$. If

$$
B(m, q)=\phi(q)^{-s} \sum_{a}^{\prime} e\left(\frac{-m a}{q}\right) \prod_{j=1}^{s} W\left(a b_{j}, \chi_{j}\right),
$$

then

$$
B(m, q)=B\left(m, q_{1}\right) B\left(m, q_{2}\right) .
$$

Proof. Apply Lemma 3.

By Lemma 1 the exceptional character $\tilde{\chi}(\bmod \tilde{r})$ is real and primitive. Then it is known $[8$, p. 159] that

$$
\tilde{r}=2^{l} p_{2} \cdots p_{t}
$$

where $p_{j}$ are distinct odd primes and $l=0$ or 2 or 3 . If $\tilde{r} \mid q$ write

$$
q=q_{1} q_{2}, \quad\left(q_{1}, q_{2}\right)=1 \text { and } q_{1}=2^{l_{1}} p_{2}^{l_{2}} \cdots p_{t}^{t_{t}}
$$

where $l_{j} \geqslant 1(j=2, \ldots, t) ; l_{1} \geqslant l$ if $l \neq 0$ and $l_{1}=0$ if $l=0$. Put

$$
B_{h}(m, q)=\phi(q)^{-s} \sum_{a}^{\prime} e\left(\frac{-m a}{q}\right) \prod_{j=1}^{s} W\left(a b_{j}, \chi_{j}^{\prime}\right) \quad(h=0,1, \ldots, s)
$$

where $\chi_{j}^{\prime}$ is defined in (4.4) and there are exactly $h \chi_{j}^{\prime}=\tilde{\chi} \chi_{0}(\bmod q)$ in the last product of (5.3). Define singular series $(h=0)$ and pseudosingular series $(h=$ $1,2, \ldots, s)$ by

$$
\mathscr{S}_{0}(m)=\sum_{q=1}^{\infty} B_{0}(m, q) \quad \text { and } \quad \mathscr{S}_{h}(m)=\sum_{\substack{q=1 \\ \tilde{r} \mid q}}^{\infty} B_{h}(m, q) .
$$

By Lemma 5(b) all series in (5.4) are absolutely convergent.

LEMMA 8. Let $\tilde{r}$ and $q_{1}$ be defined as in (5.1), (5.2). If $B_{h}\left(m, q_{1}\right) \neq 0$ then $q_{1}=d_{k} \tilde{r}$ or $2 d_{k} \tilde{r}$ where $d_{k}$ is a divisor of $k$.

Proof. For each $p_{j}(j=1, \ldots, t)$ in (5.1) with $p_{1}=2$ let $p_{j}^{\theta_{j}} \| k$. Suppose that $l_{1} \geqslant 4+\theta_{1}$ or $l_{j} \geqslant 2+\theta_{j}$ for some $j \geqslant 2$. For simplicity we only give the details for the case $j=2$. Let

$$
l_{2} \geqslant \theta_{2}+2 \text {. }
$$

Since no prime can divide all $b_{j}$, we may assume that $p_{2}+b_{1}$. Factorizing the exceptional character $\tilde{\chi}$ and the character $\chi_{1}^{\prime}$ in (5.3) we have

$$
\begin{gathered}
\tilde{\chi}(\bmod \tilde{r})=\tilde{\chi}_{1}\left(\bmod 2^{\prime}\right) \prod_{j=2}^{t} \tilde{\chi}_{j}\left(\bmod p_{j}\right), \\
\chi_{1}^{\prime}\left(\bmod q_{1}\right)=\prod_{j=1}^{t} \chi_{1 j}^{\prime}\left(\bmod p_{j}^{l_{j}}\right),
\end{gathered}
$$


where each $\chi_{1 j}^{\prime}$ is either $\chi_{0}\left(\bmod p_{j}^{l_{j}}\right)$ or $\tilde{\chi}_{j} \chi_{0}\left(\bmod p_{j}^{l_{j}}\right)$. By (3.2) for each $a$ with $\left(a, q_{1}\right)=1$ there are $a_{j}(j=1, \ldots, t)$ with $\left(a_{j}, p_{j}\right)=1$ such that $W\left(a b_{1}, \chi_{1}^{\prime}\right)=$ $\prod_{j=1}^{t} W\left(a_{j} b_{1}, \chi_{1 j}^{\prime}\right)$. Then by (5.5) and Lemma 6 with $\phi=0$, for each $a$ in $\Sigma_{a}^{\prime}$ of (5.3) we have $W\left(a_{2} b_{1}, \chi_{12}^{\prime}\right)=0$. So by (5.3) if $B_{h}\left(m, q_{1}\right) \neq 0$ then $l \leqslant l_{1} \leqslant l+1+\theta_{1}$ and $1 \leqslant l_{j} \leqslant 1+\theta_{j}(j=2,3, \ldots, s)$. This proves Lemma 8 .

LEMMA 9. (a) $\mathscr{S}_{0}(m) \gg B^{s(1-s)}$ and (b) $\mathscr{S}_{h}(m) \ll \mathscr{S}_{0}(m)(\log N)^{-1 / 2} \quad(h=$ $1,2, \ldots, s)$.

Proof. Part (a) is Lemma 5 in [9].

We come now to prove part (b). For each $q$ with $\tilde{r} \mid q$ define $q_{1}$ and $q_{2}$ as in (5.2). Since, by the hypothesis on $b_{j}$, no prime can divide more than $s-s_{0} b_{j}$, we have

$$
\prod_{j=1}^{s}\left(q_{1}, b_{j}\right) \leqslant q_{1}^{s-s_{0}} .
$$

Then by (5.3) and Lemma 5 with $\varepsilon=(10 s)^{-1}$ we have

$$
B_{h}\left(m, q_{1}\right) \ll \phi\left(q_{1}\right)^{-s+1} q_{1}^{s / 2+1 / 10} q_{1}^{\left(s-s_{0}\right) / 2} \ll q_{1}^{6 / 5-s_{0} / 2} .
$$

Then by Lemma 8 and $s_{0} \geqslant 2 k \geqslant 4$ (see (1.1)) we have

$$
B_{h}\left(m, q_{1}\right) \ll \tilde{r}^{-4 / 5} .
$$

So by Lemma 8 again we have

$$
\sum_{q_{1}=1}^{\infty} B_{h}\left(m, q_{1}\right) \ll \tilde{r}^{-4 / 5}
$$

On the other hand, by Lemma 5(a), the divisibility hypothesis on $b_{j}$ and $\left|W\left(a b_{j}, \chi_{j}^{\prime}\right)\right| \leqslant \phi\left(p^{t}\right)$, we see that the product in $B_{0}\left(m, p^{t}\right)$ in (5.3) satisfies

$$
\left|\prod_{j=1}^{s} W\left(a b_{j}, \chi_{j}^{\prime}\right)\right| \leqslant(2 k)^{s_{0}} p^{t s_{0} / 2} \phi\left(p^{t}\right)^{s-s_{0}}
$$

So by (5.3) and $s_{0} \geqslant 4$ we have

$$
\left|B_{0}\left(m, p^{t}\right)\right| \leqslant \phi\left(p^{t}\right)^{-s_{0}+1}(2 k)^{s_{0}} p^{t s_{0} / 2} \leqslant(4 k)^{s} p^{t\left(1-s_{0} / 2\right)}<c_{1} p^{-t} .
$$

For each $p$ there exists some $b_{j}=b_{1}$, say, which is not divisible by $p$. By Lemma 6 for each $a$ with $(a, p)=1$ we have $W\left(a b_{1}, \chi_{0}\right)=0$ if $t \geqslant \nu+2$ where $\nu$ is defined in (1.3) and $p^{t}$ is the modulus of $\chi_{0}$. So by (5.3) we have $B_{0}\left(m, p^{t}\right)=0$ if $t \geqslant \nu+2$. Then by Lemma 7 and $\left(\tilde{r}, q_{2}\right)=1$

$$
\begin{aligned}
\sum_{q_{2}=1}^{\infty} B_{0}\left(m, q_{2}\right) & =\prod_{p+\tilde{r}}\left(1+\sum_{t=1}^{\nu_{1}} B_{0}\left(m, p^{t}\right)\right) \\
& =\sum_{q=1}^{\infty} B_{0}(m, q) / \prod_{p \mid \tilde{r}}\left(1+\sum_{t=1}^{\nu_{1}} B_{0}\left(m, p^{t}\right)\right)
\end{aligned}
$$

where $\nu_{1}=\nu+1$. Separate the last product $\Pi_{p \mid \tilde{r}}$ into $\Pi_{p \mid \tilde{r}, p \leqslant c_{2}}$ and $\Pi_{p \mid \tilde{r}, p>c_{2}}$ where $c_{2}=4 c_{1}$. Same as that in the proof of Lemma 5 in $[9$, see (4.16) and the product $\Pi_{1}$ on p. 197] which depends essentially on (1.1)-(1.5) and the divisibility 
condition on $b_{j}$ in Theorem 1 , we have that the first product $\prod_{p \mid \tilde{r}, p \leqslant c_{2}}$ satisfies

$$
\begin{aligned}
\prod_{\substack{p \leqslant \tilde{r} \\
p \leqslant c_{2}}}\left(1+\sum_{t=1}^{\nu_{1}} B_{0}\left(m, p^{t}\right)\right) & \geqslant \prod_{\substack{p \mid \tilde{r} \\
p \leqslant c_{2}}} \phi\left(p^{\nu_{1}}\right)^{-s} p^{\nu_{1}} \\
& \geqslant \prod_{p \leqslant c_{2}} p^{\nu_{1}(1-s)}=c_{3}>0 .
\end{aligned}
$$

For the second product $\Pi_{p \mid \tilde{r}, p>c_{2}}$, by (5.7) we have

$$
\begin{aligned}
\prod_{\substack{p \mid \tilde{r} \\
p>c_{2}}}\left(1+\sum_{t=1}^{\nu_{1}} B_{0}\left(m, p^{t}\right)\right) & >\prod_{c_{2}<p \leqslant \tilde{r}}\left(1-c_{1} \sum_{t=1}^{\infty} p^{-t}\right) \\
& >\prod_{c_{2}<p \leqslant \tilde{r}}\left(1-c_{2} / 2 p\right) \gg(\log \tilde{r})^{-c_{2}} .
\end{aligned}
$$

The last inequality is a simple modification of Theorem 9.3 in [8, p. 92]. Now by (5.8), (5.9) we have

$$
\sum_{q_{2}=1}^{\infty} B_{0}\left(m, q_{2}\right) \ll \mathscr{S}_{0}(m)(\log \tilde{r})^{c_{2}}
$$

Finally, by $(5.2)$ we see that $\tilde{\chi} \chi_{0}(\bmod q)$ can be factorized as the product of $\tilde{\chi} \chi_{0}$ $\left(\bmod q_{1}\right)$ and $\chi_{0}\left(\bmod q_{2}\right)$. Then by (5.4), Lemma 7, (5.6), (5.10) we have

$$
\mathscr{S}_{h}(m)=\sum_{q_{1}=1}^{\infty} B_{h}\left(m, q_{1}\right) \sum_{q_{2}=1}^{\infty} B_{0}\left(m, q_{2}\right) \ll \mathscr{S}_{0}(m)(\log N)^{-1 / 2}
$$

since by (3.1) we have

$$
\tilde{r}^{4 / 5}(\log \tilde{r})^{-c_{2}} \gg(\log N)^{1 / 2}
$$

This proves Lemma 9.

6. Major arcs. II.

LEMMA 10. We have

$$
\int_{(q Q)^{-1}}^{1 / 2}\left|\prod_{j=1}^{s} I^{\prime}\left(b_{j} \eta\right)\right| d \eta \ll(q Q)^{s-1} N^{s(1-k)}
$$

where $I^{\prime}\left(b_{j} \eta\right)$ is defined in (4.5).

Proof. If $0<\eta \leqslant 1 / 2$ then for any $n \geqslant 1$ we have $\sum_{l=0}^{n} e(l \eta) \ll|\eta|^{-1}$. Let $\phi=1 / k$ or $\tilde{\beta} / k$. Then by Abel's partial summation formula and (2.6)

$$
\begin{aligned}
b^{\phi} I^{\prime}(b \eta) & \ll|\eta|^{-1}\left\{\left|b N^{k}\right|^{\phi-1}+\int_{|b| G^{k}}^{|b| N^{k}}\left|\frac{d}{d y} y^{\phi-1}\right| d y\right\} \\
& \ll|\eta|^{-1}\left(|b| G^{k}\right)^{\phi-1} \ll|\eta|^{-1} N^{1-k} .
\end{aligned}
$$

So the lemma follows.

Let

$$
J_{h}(m)=\int_{-1 / 2}^{1 / 2} \prod_{j=1}^{h} \tilde{I}\left(b_{j} \eta\right) \prod_{j=h+1}^{s} I\left(b_{j} \eta\right) e(-m \eta) d \eta \quad(h=0,1, \ldots, s) .
$$


LEMMA 11. (a) $\left|J_{h}(m)\right| \leqslant J_{0}(m)(h=1,2, \ldots, s)$.

(b) If

$$
|m| \leqslant(N / 4)^{k} s^{-1}
$$

then

$$
J_{0}(m) \gg B^{-s / k} N^{s-k} .
$$

Proof. Part (a) follows from (6.1) and part (b) is essentially Lemma 8 [9].

We come now to treat those terms in category (B) defined in $\$ 4$. In category (B) we choose $\prod_{j=1}^{h} \tilde{\mathscr{I}}_{j} \prod_{j=h+1}^{s} \mathscr{I}_{j} \quad(h=0,1, \ldots, s)$ to represent those terms $\prod_{j=1}^{s} \mathscr{I}_{j}^{\prime}$ having exactly $h$ factors $\tilde{\mathscr{I}}_{j}$. Put

$$
\begin{gathered}
T_{0}(m)=\sum_{q \leqslant P} \sum_{a}^{\prime} e\left(\frac{-m a}{q}\right) \int_{-\delta_{q}}^{\delta_{q}}\left(\prod_{j=1}^{s} \mathscr{I}_{j}\right) e(-m \eta) d \eta, \\
\tilde{T}_{h}(m)=\sum_{\substack{q \leqslant P \\
\tilde{r} \mid q}} \sum_{a}^{\prime} e\left(\frac{-m a}{q}\right) \int_{-\delta_{q}}^{\delta_{q}}\left(\prod_{j=1}^{h} \tilde{\mathcal{I}}_{j} \prod_{j=h+1}^{s} \mathscr{I}_{j}\right) e(-m \eta) d \eta \\
\quad(h=1,2, \ldots, s) .
\end{gathered}
$$

By (4.1), $s \geqslant 5$, Lemmas 10 and 5 with $\varepsilon=(10 s)^{-1}$ we have

$$
\begin{gathered}
\sum_{\substack{q \leqslant P \\
\tilde{r} \mid q}} \sum_{a}^{\prime} e\left(\frac{-m a}{q}\right) \int_{\delta_{q}}^{1 / 2}\left(\prod_{j=1}^{h} \tilde{\mathcal{I}}_{j} \prod_{j=h+1}^{s} \mathscr{I}_{j}\right) e(-m \eta) d \eta \\
\ll N^{s(1-k)} Q^{s-1} \sum_{q \leqslant P} \phi(q)^{-s+1}\left(\prod_{j=1}^{s}\left(q, b_{j}\right)^{1 / 2} q^{1 / 2+\varepsilon}\right) q^{s-1} \\
\ll N^{s-k} B^{s / 2} P^{-3 / 10}=E_{2}, \quad \text { say. }
\end{gathered}
$$

So, if we replace the integral $\int_{-\delta_{q}}^{\delta_{q}}$ in (6.3) by $\int_{-1 / 2}^{1 / 2}$ we have the error $E_{2}$ given in (6.4). Then by (6.1), (6.3), (4.1) we have

$$
\begin{aligned}
\tilde{T}_{h}(m)= & J_{h}(m)\left\{\sum_{\substack{q \leqslant P \\
\tilde{r} \mid q}} \phi(q)^{-s} \sum_{a}^{\prime} e\left(\frac{-m a}{q}\right) \prod_{j=1}^{h} W\left(a b_{j}, \tilde{\chi} \chi_{0}\right) \prod_{j=h+1}^{s} W\left(a b_{j}, \chi_{0}\right)\right\} \\
& +E_{2} .
\end{aligned}
$$

Similarly, by Lemma 5 , if we replace the sum $\sum_{q<P, \tilde{r} \mid q}$ in (6.5) by $\sum_{q=1, \tilde{r} \mid q}^{\infty}$ we have an error $\ll B^{s / 2} P^{-3 / 10}$. So by (5.4), (6.5) we have

$$
\tilde{T}_{h}(m)=J_{h}(m)\left(\mathscr{S}_{h}(m)+E_{3}\right)+E_{2} \quad(h=1,2, \ldots, s),
$$

where $E_{3}=O\left(E_{2} N^{k-s}\right)$. By the same argument we have

$$
T_{0}(m)=J_{0}(m)\left(\mathscr{S}_{0}(m)+E_{3}\right)+E_{2} \text {. }
$$

Note that each representative in either category (A) or (B) defined in $\$ 4$ represents at most $O(1)$ terms. It follows from (4.2), (4.10), (6.7), (6.6) that

$$
\begin{aligned}
R_{1}(m)= & J_{0}(m)\left(\mathscr{S}_{0}(m)+E_{3}\right)+O\left(\sum_{h=1}^{s} J_{h}(m)\left\{\mathscr{S}_{h}(m)+E_{3}\right\}\right) \\
& +O\left(E_{2}+E_{1}\right) .
\end{aligned}
$$


By (4.10), (6.4), Lemmas 9(a) and 11(b) we see that for $j=1,2$

$$
E_{3} / \mathscr{S}_{0}(m) \text { and } E_{j} / J_{0}(m) \mathscr{S}_{0}(m) \ll B^{2 s^{2}} P^{-3 / 10} .
$$

It follows from (6.8), (6.9), (2.1), Lemmas 11(a) and 9(b) that

$$
R_{1}(m)>\frac{1}{2} J_{0}(m) \mathscr{S}_{0}(m) \text {. }
$$

\section{Minor arcs.}

LeMma 12. If $\alpha \in m$ then

$$
\sum_{p \leqslant N} e\left(\alpha b p^{k}\right) \ll N|b| P^{-\omega(k)}
$$

where $\omega(k)^{-1}=4^{(k+2)}(k+1)$.

Proof. This is essentially Lemma 11 [9] (see also Lemma 5 [1]).

Let

$$
R_{2}(m)=\int_{m} \prod_{j=1}^{s} S\left(b_{j} \alpha\right) e(-m \alpha) d \alpha .
$$

Then by Lemma 12 and the same argument as Lemma 12 in [9] we have

$$
R_{2}(m) \ll N^{s-k} B^{s} P^{-\omega(k)}(\log N)^{c_{4}} .
$$

By (4.2), (6.10), Lemmas 9(a), 11(b) and (2.1)

$$
\begin{gathered}
\int_{Q^{-1}}^{1+Q^{-1}} \prod_{j=1}^{s} S\left(b_{j} \alpha\right) e(-m \alpha) d \alpha=R_{1}(m)+R_{2}(m) \\
\gg N^{s-k} B^{-s^{2}}\left\{1-c_{5} B^{s(s+1)} P^{-\omega(k)}(\log N)^{c_{4}}\right\}>0 .
\end{gathered}
$$

Choose the least $N$ satisfying (2.1) and (6.2). So (7.1) implies the existence of a solution of $\sum_{j=1}^{s} b_{j} p_{j}^{k}=m$ in primes $p_{j}$ and

$$
\max _{1 \leqslant j \leqslant s} p_{j} \leqslant N \leqslant C_{1}(k) m^{1 / k}+C_{2}(k)^{(\log B)^{2}} .
$$

This completes the proof of Theorem 1.

RemarK. Combining the Circle Method with the Sieve Method, when $k=1$ and $s=3$, the author [10] is able to obtain a bound for solutions of (1.6) to be $B^{A}$ where $A>0$ is an absolute constant. However, for $k \geqslant 2$ it seems that these two methods do not combine well to replace the $(\log B)^{2}$ in (1.7) by $\log B$.

\section{REFERENCES}

1. A. Baker, On some diophantine inequalities involving primes, J. Reine Angew. Math. 228 (1967), $166-181$.

2. B. J. Birch and H. Davenport, Quadratic equations in several variables, Proc. Cambridge Philos. Soc. 54 (1958), 135-138.

3. J. W. S. Cassels, Bounds for the least solutions of homogeneous quadratic equations and addendum to the same, Proc. Cambridge Philos. Soc. 51 (1955), 262-264; ibid. 52 (1956), 604.

4. H. Davenport, Multiplicative number theory, 2nd ed., Graduate Texts in Math., no. 74, SpringerVerlag. Berlin and New York, 1980.

5. P. X. Gallagher, A large sielle density' estimate near $\sigma=1$, Invent. Math. 11 (1970), 329-339. 
6. H. Hasse, Vorlesungen über Zahlentheorie, Grundlehren. Math. Wiss., Band 59, Springer-Verlag, Berlin and New York, 1964.

7. L. K. Hua, Additive theory of prime numbers, Transl. Math. Mono., vol. 13, Amer. Math. Soc., Providence, R. I., 1965.

8. Introduction to number theory, Springer-Verlag, Berlin and New York, 1982.

9. M. C. Liu, Bounds for prime solutions of some diagonal equations, J. Reine Angew. Math. 332 (1982), $188-203$.

10. M. C. Liu, An improved bound for prime solutions of some ternary equations, preprint.

11. J. Pitman and D. Ridout, Diagonal cubic equations and inequalities, Proc. Roy. Soc. (A) 297 (1967), 476-502.

12. J. Pitman, Bounds for solutions of diagonal equations, Acta Arith. 19 (1971), 223-241.

Department of Mathematics, University of Hong Kong, Hong Kong 